\title{
SYMPOSIUM \\ ANESTHESIE ET GLANDES ENDOCRINES
}

ChATEAU FRONTENAC, QUÉBEC, P.Q, DIMANCHE, LE 27 JUIN 1971

\section{Programme}

$8 \mathrm{~h}$ à $8 \mathrm{~h} 30$ Inscription

8 h 30 à 8 h 45 Introduction : Le Président - Raynald Déry

8 h 45 à 9 h 30 "Influence des agents anesthésiques sur l'activité neuroendocrinienne"

Leroy D. Vandam, Professeur, Harvard Medical School, Boston

9 h 30 à 10 h 15 "Le Système Rénine-Angiotensine-Aldostérone; l'aldostéronisme primaire et l'anesthésie de l'hypertendu"

Jacques Genest, Professeur et Directeur scientifique de l'Institut de Recherches cliniques de Montréal

$10 \mathrm{~h} 15$ à $10 \mathrm{~h} 45$ Pause-café

10 h 45 à 11 h 30 "Les glucocorticoïdes en Anesthésie"

Tsutomu Oyama, Professeur, Faculté de Médecine de l'Université Hirosaki, Japon

11 h 30 à 12 h 15 "Physiopathologie du système adrénergique en anesthésieréanimation. Déductions thérapeutiques"

Pierre Huguenard, Professeur, CHU Henri-Mondor, Paris

12 h 15 à 13 h 45 Déjeuner

$13 \mathrm{~h} 45$ à 14 h 30 "Phéochromocytome et anesthésie"

Allen B. Dobkin, Professeur, State University of New York, Syracuse, New York

$14 \mathrm{~h} 30$ à 15 h 15 "L'anesthésie et le métabolisme des hydrates de carbonne"

Robert G. Merin, Professeur agrégé, The University of Rochester Medical Center, New York

$15 \mathrm{~h} 15$ à $15 \mathrm{~h} 45$ Pause-café

15 h 45 à 16 h 15 "Les autocoïdes en Anesthésie"

Raynald Déry, Professeur agrégé, Université Laval, Québec

$16 \mathrm{~h} 15$ à $17 \mathrm{~h} 15$ Discussion et Questions 